University of Windsor

Scholarship at UWindsor

1975

\title{
Comments on a new mathematical technique in the theory of complex spectra
}

J Drake

Gordon W. F. Drake

University of Windsor

M. Schlesinger

Follow this and additional works at: https://scholar.uwindsor.ca/physicspub

Part of the Physics Commons

\section{Recommended Citation}

Drake, J; Drake, Gordon W. F.; and Schlesinger, M.. (1975). Comments on a new mathematical technique in the theory of complex spectra. Journal of Physics B: Atomic and Molecular Physics, 8 (7), 1009-1015. https://scholar.uwindsor.ca/physicspub/44

This Article is brought to you for free and open access by the Department of Physics at Scholarship at UWindsor. It has been accepted for inclusion in Physics Publications by an authorized administrator of Scholarship at UWindsor. For more information, please contact scholarship@uwindsor.ca. 
Comments on a new mathematical technique in the theory of complex spectra

This article has been downloaded from IOPscience. Please scroll down to see the full text article.

1975 J. Phys. B: At. Mol. Phys. 81009

(http://iopscience.iop.org/0022-3700/8/7/007)

View the table of contents for this issue, or go to the journal homepage for more

Download details:

IP Address: 137.207.184.30

The article was downloaded on 26/04/2013 at 15:45

Please note that terms and conditions apply. 


\title{
Comments on a new mathematical technique in the theory of complex spectra $\dagger$
}

\author{
Judith Drake, G W F Drake $\ddagger$ and M Schlesinger $\$$ \\ Department of Physics, University of Windsor, Windsor, Ontario, Canada N9E 3P4
}

Received 11 December 1974

\begin{abstract}
A large body of work on the algebraic properties of the Gelfand labelling scheme for atoms with several equivalent electrons has recently been synthesized by Harter into a compact procedure for the construction of total angular momentum eigenfunctions and the evaluation of angular coefficients. In this paper, certain ambiguities in the procedure are removed. Also, an improved method for the diagonalization of the angular momentum matrix in the Gelfand basis set is presented. As an example, the doublet states of the $f^{3}$ configuration are discussed.
\end{abstract}

\section{Introduction}

A recent paper by Harter (1973) has drawn attention to an elegant alternative to the usual Racah scheme for the construction of angular momentum eigenfunctions of complex atoms with several equivalent electrons. Instead of the usual fractional parentage coefficients, seniority quantum numbers, etc, the scheme described by Harter starts from the triangular 'Gelfand pattern' for labelling a complete set of many-particle angular momentum functions. The Gelfand labelling corresponds to a canonical chain of unitary groups $U(n) \supset U(n-1) \supset \ldots \supset U(1)$ for $n$ equivalent electrons. Each Gelfand pattern can be converted uniquely into a more compact Young Tableau representation. Each Young Tableau corresponds to an antisymmetrized $n$-particle state with definite total spin $S$ and component of total angular momentum $M$ in the $z$ direction, but is a mixture of states with all possible values of $L$. The total angular momentum eigenfunctions of definite spin are found by diagonalizing the matrix representation of $L^{2}$ in each $S, M$ subspace. The diagonalization is particularly simple since one knows in advance that the eigenvalue must be $M(M+1),(M+1)(M+2)$, $\ldots L_{\max }\left(L_{\max }+1\right)$ with some eigenvalues occurring more than once.

To carry through the above calculation, it is necessary to have an algorithm for the evaluation of the matrix elements of irreducible tensor operators in the Young tableau representation. Algebraic formulae in terms of the original Gelfand patterns are reviewed by Louck (1970 see also references contained therein). Harter gives a diagrammatic counting representation (the so-called jawbone formula) of the equivalent formulae in terms of Young tableaux. Although the jawbone diagram provides a convenient and rapid method of evaluating matrix elements, Harter's presentation contains ambiguities which are difficult to resolve without reference to the algebraic formulae.

$\dagger$ Research supported by the National Research Council of Canada.

$\ddagger$ Alfred P Sloan Foundation Fellow.

$\S$ On sabbatical leave $(1974 / 1975)$ at the Clarendon Laboratory, University of Oxford, UK. 
In this paper, we give an amplification of Harter's jawbone diagram to resolve the ambiguities. We also describe a method for finding the eigenvectors of $L^{2}$ which does not require any explicit matrix diagonalizations or inversions. The entire computational scheme has been programmed and an example involving the doublet states of three equivalent $\mathrm{f}$ electrons is presented.

\section{Outline of theory}

The reader is referred to Harter's paper (figures 1 and 6) for an easily understood description of the procedure for constructing Gelfand arrays and converting them to Young tabelaux. Consider a configuration containing $n$ equivalent electrons each with angular momentum $l$. The Young tableaux all have $n$ boxes arranged into two columns in the form

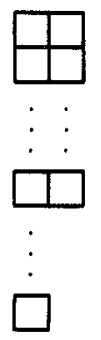

Each box is labelled by an integer $\mu=1,2,3, \ldots, 2 l+1$ corresponding to the one-electron orbital magnetic quantum numbers $m, m-1, \ldots,-m$ respectively, such that no column contains the same integer more than once. The spin multiplicity of each possible tableau is $2 S+1$, where $2 S$ is the number of unpaired boxes, and the $M$ quantum number is $n(l+1)$ minus the sum of the $n$ numbers labelling the boxes.

The fundamental rule for calculating the matrix elements of irreducible unit tensor operators between Young tableaux is as follows. Define single particle operators $E_{\mu, \mu^{\prime}}$ to have the effect of changing a box labelled by $\mu^{\prime}$ into a box labelled by $\mu$. The matrix elements of $E_{\mu, \mu^{\prime}}$ in a space of single particle states are

$$
\left\langle v\left|E_{\mu, \mu^{\prime}}\right| \lambda\right\rangle=\delta_{\mu, \nu} \delta_{\mu^{\prime}, \lambda} \quad v, \lambda=1,2, \ldots, 2 l+1
$$

Using the Wigner-Eckart theorem a unit irreducible operator $V_{q}^{k}$ can then be written in the form

$$
V_{q}^{k}=\sum_{\mu, \mu^{\prime}}^{2 l+1}(-1)^{l-m}(2 k+1)^{1 / 2}\left(\begin{array}{ccc}
l & k & l \\
-m & q & m^{\prime}
\end{array}\right) E_{\mu, \mu^{\prime}}
$$

for a single-electron state with angular momentum $l$. Here, $m=l-\mu+1$ and $m^{\prime}=l-\mu^{\prime}+1$. For an $n$-particle state, if $V_{q}^{k}=\Sigma_{i=1}^{n} V_{q}^{k}(i)$, then $E_{\mu, \mu^{\prime}}$ in (2) is understood to be a sum of operators, one for each of the $n$ boxes. The matrix elements of $E_{\mu, \mu^{\prime}}$ are more complicated for the $n$-particle case. If the $i$ th box is labelled by $\mu-1$, then $E_{\mu, \mu-1}(i)$ changes the label to $\mu$, but the numerical factor, instead of being unity as in (1), is computed from Harter's jawbone counting diagram shown in figure 1. Each of the four diagrams under the square root corresponds to an integer factor which is the product of all the arrow lengths (hook lengths). In general, there is one arrow for each row. The arrows are drawn unambiguously according to the following rules : 
(o)

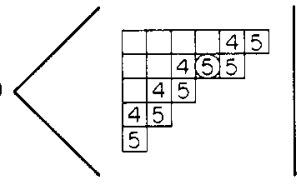

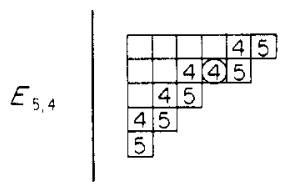

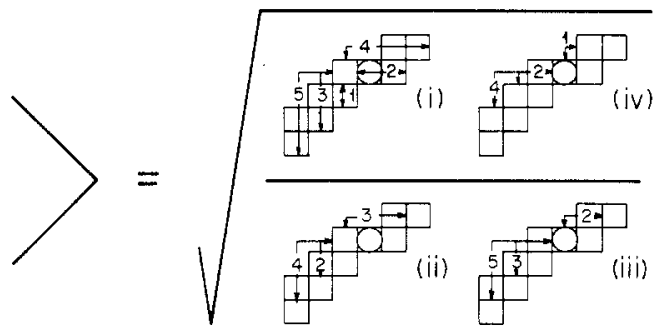

$=\sqrt{\frac{4}{3}}$

(b)<smiles>CCCCCC</smiles>

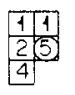

$E_{5,4}$
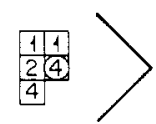

$=$

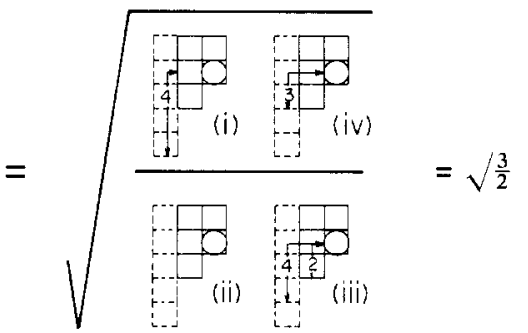

Figure 1. Examples of the jawbone counting diagram for the case $\mu=5$. The Roman numerals correspond to the rules stated in the text. Diagram $b$ (ii), for which no arrows can be drawn according to rule (ii), contributes a factor of unity.

(i) Starting from the box to the left of the $i$ th box, draw all arrows to pass through the last box labelled $\mu$ in each row.

(ii) Starting from the box to the left of the $i$ th box, draw all arrows through the box to the left of the first box labelled $\mu$ in each row.

(iii) Starting from the $i$ th box, draw all arrows through the last box labelled $\mu-1$ in each row.

(iv) Starting from the $i$ th box, draw all arrows through the box to the left of the first box labelled $\mu-1$ in each row.

In drawing the arrows, a column of length $2 l+1$ can be added to the left side of each of the four tableaux without changing the numerical value. This is sometimes necessary to ensure that all contributions are counted. Arrows that cannot be drawn contribute a factor of unity. Although the rules are lengthy to state, they can be programmed into a compact and efficient computer routine.

The matrix elements of $E_{\mu-1, \mu}$ can be obtained from those of $E_{\mu, \mu-1}$ by use of the property

$$
\left\langle\nu\left|E_{\mu-1, \mu}\right| \lambda\right\rangle=\left\langle\lambda\left|E_{\mu, \mu-1}\right| v\right\rangle
$$

where $v$ and $\lambda$ denote complete tableaux of $n$ boxes. The matrix elements for $|\Delta \mu|>1$ can be calculated from those for $|\Delta \mu|=1$ by the use of algebraic relations among the $E_{\mu, \mu^{\prime}}$ operators (or their matrix representations in a particular basis set of Young tableaux) of the form

$$
E_{\mu, \mu^{\prime}}=E_{\mu, \lambda} E_{\lambda, \mu^{\prime}}-E_{\lambda, \mu^{\prime}} E_{\mu, \lambda}, \quad\left(\mu \neq \mu^{\prime}\right) .
$$

Relations of this type can easily be verified in a basis set of completely antisymmetric wavefunctions. For this case, the Young tableaux are single columns of $n$ boxes and the 
matrix elements, which are all zero or unity, can be written down by inspection. As an example of (4),

$$
E_{1,3}=E_{1,2} E_{2,3}-E_{2,3} E_{1,2} .
$$

Thus, all the $E_{\mu, \mu^{\prime}}$ can be determined by a sequence of matrix multiplications once the $E_{\mu, \mu \pm 1}$ are calculated by the jawbone diagram. Large amounts of computer storage are not required since most of the matrix elements are zero. The diagonal matrix elements $\left\langle v\left|E_{\mu, \mu}\right| v\right\rangle$ equal the number of times the label $\mu$ occurs in the tableau $v$.

\section{Example involving the doublet states of the configuration $\mathbf{f}^{3}$}

Consider all possible states of the type $\mathrm{f}^{32} \mathrm{~L}$. The possible Young tableaux generated from the Gelfand labelling scheme are arranged in table 1 according to the value of $M$. It is immediately obvious from the number of tableaux in each row that the number of states with $L=8,7, \ldots, 0$ is $1,1,1,2,2,2,2,1,0$ respectively, in agreement with the enumeration of Wybourne (1970). As suggested by Harter, the eigenvectors $\psi_{L M}$ of

$$
L^{2}=\left(-L_{1}^{1} L_{-1}^{1}-L_{-1}^{1} L_{1}^{1}+L_{0}^{1} L_{0}^{1}\right)
$$

can be calculated directly by diagonalizing the matrix representation of $L^{2}$ in each subset of tableaux with the same $M$ shown in table 1 . A much simpler and more efficient procedure requiring only a small number of matrix multiplications is described below.

Let the tableaux in table 1 be labelled sequentially by (1), (2), . from the left to the right across each row and from the top down. Since there is only a single tableau for $M=8$, it is clear that

$$
\psi_{8,8}=(1)
$$

The lowering operator $L_{-1}^{1}$ connects the tableaux in each row with the ones in the row beneath. $L_{-1}^{1}$ is related to $V_{-1}^{1}$ in (2) by

$$
L_{-1}^{1}=V_{-1}^{1}\langle l\|L\| l\rangle
$$

with

$$
\langle l\|L\| l\rangle=\frac{[l(l+1)(2 l+1)]^{1 / 2}}{\sqrt{3}} .
$$

Using the jawbone counting diagram, the first few submatrices of $L_{-1}^{1}$ are

$$
\begin{aligned}
& L_{-1}^{1}(8 \rightarrow 7)=-\left(\begin{array}{cc}
\sqrt{3} \\
\sqrt{ } 5
\end{array}\right) \\
& (2) \\
& (3) \\
& L_{-1}^{1}(7 \rightarrow 6)=-\left(\begin{array}{rr}
\sqrt{\frac{5}{2}} & \sqrt{ } 6 \\
\sqrt{\frac{15}{2}} & 0 \\
0 & \sqrt{6}
\end{array}\right)
\end{aligned}
$$




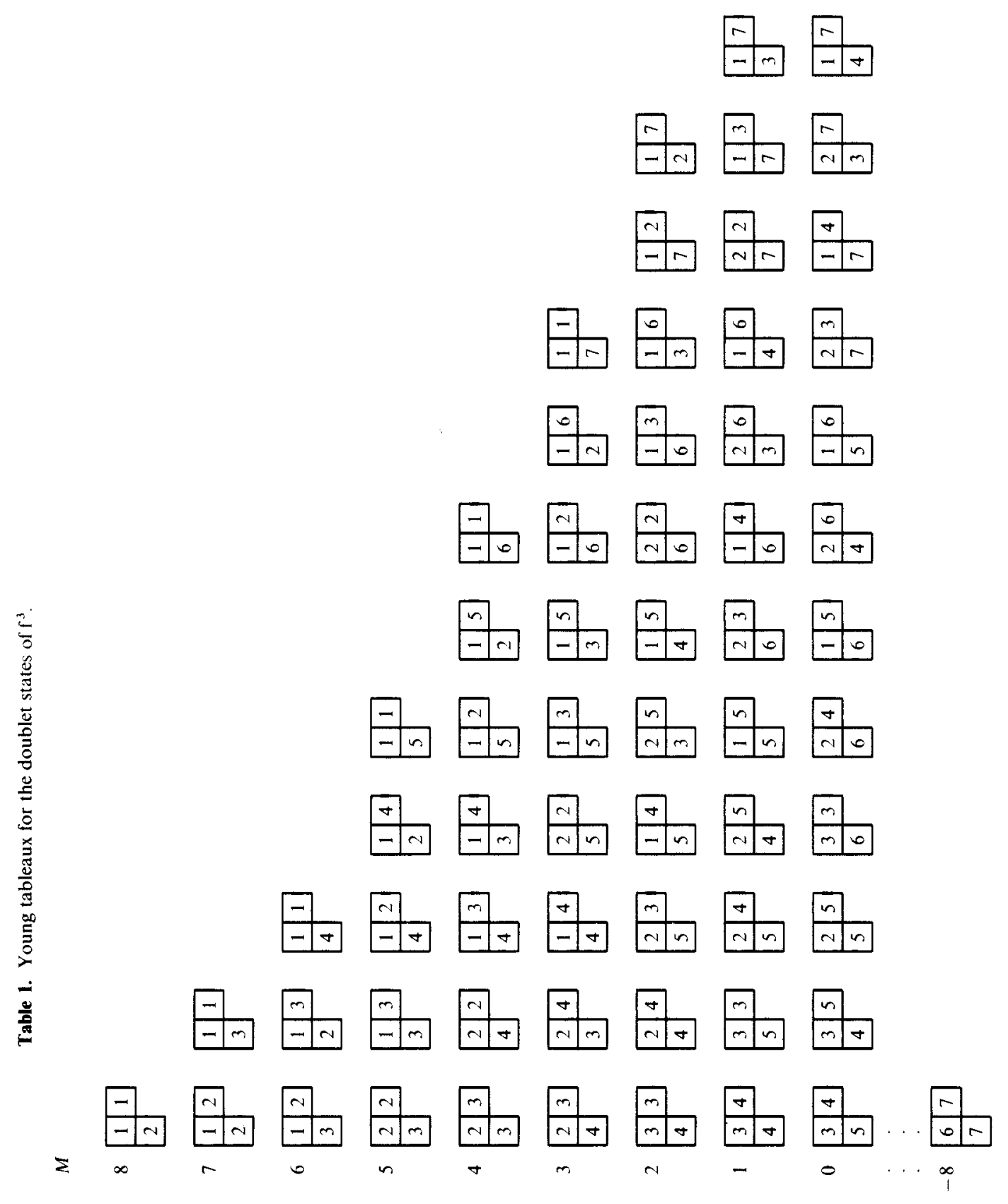




$$
L_{-1}^{1}(6 \rightarrow 5)=-\left(\begin{array}{rrr}
(4) & (5) & (6) \\
\sqrt{ } 6 & 0 & 0 \\
\sqrt{\frac{5}{2}} & \sqrt{\frac{15}{2}} & 0 \\
\sqrt{6} & 0 & \sqrt{6} \\
0 & \sqrt{6} & 0 \\
0 & 0 & \sqrt{6}
\end{array}\right)
$$

The eigenvector $\psi_{L, M-1}$ is then obtained lrom $\psi_{L, M}$ by means of the well known formula

$$
\psi_{L, M-1}=\left(\frac{2}{(L+M)(L-M+1)}\right)^{1 / 2} L_{-1}^{1} \psi_{L, M} .
$$

For example

$$
\begin{aligned}
\psi_{8,7} & =\frac{1}{\sqrt{8}} L_{-1}^{1}(8 \rightarrow 7) \psi_{8,8} \\
& =\frac{-1}{\sqrt{8}}\left(\begin{array}{l}
\sqrt{3} \\
\sqrt{ } 5
\end{array}\right)
\end{aligned}
$$

In this way, all states $\psi_{L, M}, L=M+1, M+2, \ldots, L_{\max }$ in the $M$ th row of table 1 can be generated from the states in the $(M+1)$ th row. The remaining (possibly multiple) state $\psi_{M, M}$ can be constructed by a projection operator technique. First, calculate the projection operator

$$
\boldsymbol{Q}_{M}=\sum_{L=M+1}^{L_{\max }} \psi_{L, M} \psi_{L, M}^{\dagger}
$$

including all multiple states in the sum. Any column of the complementary operator $\boldsymbol{P}_{M}=1-\boldsymbol{Q}_{M}$ is then an unnormalized eigenvector $\psi_{M, M}$. Let us arbitrarily choose the first colurnn. If a second state $\psi_{M M}^{\prime}$ exists, then the procedure is repeated by calculating

$$
\boldsymbol{Q}_{M}^{\prime}=\boldsymbol{Q}_{M}+\psi_{M, M} \psi_{M, M}^{\dagger}
$$

with $\psi_{M, M}$ normalized to unity. The second column of $\boldsymbol{P}^{\prime}=1-\boldsymbol{Q}^{\prime}$ is an orthogonal state $\psi_{M, M}^{\prime}$. The procedure is continued until the basis set is complete, ie $\boldsymbol{P}^{\prime} \equiv 0$. Any linear combination of states $\alpha \psi_{L, M}+\beta \psi_{L, M}^{\prime}$ is, of course, also an eigenvector. The use of the lowering formula (9) guarantees the same linear combination and a definite phase relationship for different values of $M$. This is not true when general matrix diagonalization techniques are used to diagonalize $L^{2}$ in each subspace. To save time, only the first $k$ columns of $Q$ and $P$ need be calculated, where $k$ is the number of times the state $\psi_{M, M}$ occurs. The $L^{2}$ matrix is not needed at all. The first few eigenvectors are

$$
\begin{aligned}
& \psi_{8,8}=(1) \\
& \psi_{8,7}=-(1 / \sqrt{ } 8)(\sqrt{ } 3, \sqrt{ } 5) \\
& \psi_{7,7}=(1 / \sqrt{ } 8)(-\sqrt{ } 5, \sqrt{ } 3) \\
& \psi_{8,6}=(1 / 4)(3, \sqrt{ } 3,2) \\
& \psi_{7,6}=(1 / \sqrt{ } 112)(-1,5 \sqrt{ } 3,-6) \\
& \psi_{6,6}=(1 / \sqrt{ } 21)(3,-\sqrt{3},-3)
\end{aligned}
$$




$$
\begin{aligned}
& \psi_{8,5}=-(1 / \sqrt{ } 168)(3 \sqrt{ } 3,3 \sqrt{ } 5,5 \sqrt{ } 3,3,2 \sqrt{ } 3) \\
& \psi_{7,5}=-(1 / \sqrt{ } 728)(-\sqrt{ } 3,7 \sqrt{ } 5,-7 \sqrt{ } 3,15,-6 \sqrt{ } 3) \\
& \psi_{6,5}=-(1 / \sqrt{ } 7)(\sqrt{3}, 0,0,-1,-\sqrt{ } 3) \\
& \psi_{5,5}=-(1 / \sqrt{ } 3367)(37 ;-4 \sqrt{ } 15,-27,10 \sqrt{ } 3,27) \\
& \psi_{5,5}^{\prime}=-(1 / \sqrt{ } 111)(0,6,-\sqrt{ } 15,-3 \sqrt{ } 5, \sqrt{ } 15)
\end{aligned}
$$

as can easily be verified by hand calculation. The numbers multiply the corresponding tableaux with the same $M$ in table 1 .

\section{Discussion}

The methods discribed by Harter and the present work provide a simple, easily programmed algorithm for the construction of angular momentum eigenfunctions in atoms containing several equivalent electrons. A knowledge of group theory is not necessary for its application. Equation (2) and the jawbone counting diagram allows the calculation of the angular coefficients for the matrix elements of any spin-independent irreducible tensor operator in the Young tableaux basis set. The computational scheme is not yet complete since it still lacks a prescription for the evaluation of spin-dependent matrix elements. Further work on this problem is in progress.

\section{References}

Harter W G 1973 Phys. Rev. A 8 2819-27

Louck J D 1970 Am. J. Phys. 38 3-42

Wybourne B G 1970 Symmetry Principles and Atomic Spectroscopy (New York: Wiley) p 73 